\title{
Light Travels in Coronal Ocean, not in Vacuum
}

\author{
Salah Eid \\ Faculty of Arts, Suez Canal University, Ismailia, EGYPT
}

\begin{abstract}
Our Earth is immersed into an "ocean" of free energetic electrons from the last layer in the atmosphere of the Sun called corona which is located above its other two layers: the cromosphere and the ionosphere. When electrons are aroused from their atoms striking the mentioned ocean of coronal electrons, then radio waves are created. Therefore light does not travel through vacuum contradicting the statement of two great figures: Maxwell and Einstein. Also, we must re-consider what is known as electromagnetic wave when we speak about light, as magnetic field surrounds only the passage of an electric current, where circles of electrons are created in the coronal ocean as circles of water are created around the passage of a stone thrown in a lake, but such magnetic field does not extend to radio waves.
\end{abstract}

Keywords: corona, electron, the speed of light, vacuum , Maxwell's celebrated equation, special relativity , Edison's achievements.

DOI: $10.7176 /$ APTA/75-04

\section{Introduction}

Solar corona is the last layer in the structure of the Sun, it is a plasma state where electrons are free from protons. In the Sun as a hydrogen star, the distance between electron and proton is the key for understanding its structure. In Sun's core where gravity is at its strongest force the distance between these two particles is at its shortest value, this fact is confirmed by Oliver Manuel's discovery a small neutron star in the core of the $\operatorname{Sun}^{(1)}$. On the other hand the last stage in the Sun's structure above its hydrogen surface its atmosphere composing mainly of three layers : the ionosphere, the chromosphere, and the corona ${ }^{(2)}$. With the distance from the center of the Sun the distance between the electron and proton gradually increases till at the last layer called corona that extends to millions of kilometers, or according to NASA's Cosmos it extends several solar radii ${ }^{(3)}$, where electrons are free from protons forming a great electronic ocean. It is important here to notice that it had become clear that radio noise storms and radio metric type II radio bursts had solar origins caused by coronal shock waves ${ }^{(4)}$. This confirms greatly our view about the source and nature of radio waves.

\section{Light as a wave:}

A wave is a circular motion with its two opposite halves, being it is the motion of one thing or millions of things, this can be applied on one electron revolving a proton, or a great number of electrons forming a "sea" of electrons like the last layer of the Sun. This ocean of free energetic electrons when interact with the gaseous atmosphere of the Earth, during its daily motion around its axis, the light of the day takes place in half of the Earth's ball ${ }^{(3)}$, and without this ocean of free energetic coronal electrons no wireless communications can occur on the Earth or outside it.

\section{Light and vacuum:}

When Michelson and Morley proved by their well known experiment that there is nothing called ether as the medium conveying the waves of light, this was a shocking result because no wave propagation without medium was known before. Einstein in 1905 based the special theory of relativity on that the speed of light $c$ takes place in vacuum and this speed is the same for all observers regardless of the motion of the light source. In fact, in 1864 the propagation of light through empty space was also Maxwell's view. ${ }^{(4)}$ With Einstein, there is no surprise about the light travelling in vacuum, as he in general relativity made the empty space curves around the Sun for the planets to revolve ${ }^{(5)}$ ! But how "nothing" can be a thing having waves like water, and can be bent as any rigid matter ?!

In fact, we have now the key for understanding the propagation of light, this key was not almost known in the days of Maxwell or Einstein, it is the mentioned ocean or stream of the coronal electrons into which again our Earth is immersed. The radiation from any source will not travel by itself on the Earth or from it to any place outside it in the universe, but it will activate this ocean of electrons of the solar corona, and the excited electrons from an atom will cause the surround coronal electrons to move with the same speed. Even when lightening any electrical bulb, the excited electrons inside the bulb will excite the coronal electrons around it, and these will affect our eyes causing the sensation of what we call vision. Although Thomas Edison said nothing about the coronal electrons, he proved that electrons would fly far from their home atoms' orbit when excited, strike "other" electrons causing them to be excited too ${ }^{(6)}$, ( and now we know that these other electrons are coronal ones) this exactly is the principal of radio waves which have specific intervals making the exciter burst with energy at specific intervals 
measured in hertz, the burst - in words of Thomas Edison- is just like an ocean wave with its high and low points. ${ }^{(7)}$. Edison therefore was the real discoverer of radio waves, although he referred to them as a sort of ether. In New York Times Des.3,1875 we read the following : an ingenious person of telegraphic antecedents has just informed the world that he has discovered a new force, which he calls the 'etheric force' ....this force, he assures us, is neither electricity nor magnetism, although it seems to be a sort of second cousin of the one. this ingenious person was in fact Thomas Edison, and the "etheric force" he discovered was in fact radio waves. (See hyperlink for much more on this). ${ }^{(8)}$ It is clear now that Edison's words seem as if they describe what we call now the coronal ocean over helming our Earth.

\section{Magnetism in light}

We accustomed to speak about light as an electromagnetic phenomenon since Oersted found that an electric current creates magnetic field, Maxwell's celebrated equation $c^{2}=\frac{1}{\varepsilon_{0} \mu_{0}}$ made the connection between electricity and magnetism an unquestionable fact, but with Oersted discovery it is clear that the passage of electric current in the mentioned ocean of coronal electrons creates circles around it like those created by a stone thrown in a lake where every point in the circle is directed to its center, this is the explanation of the magnetic field around the electric current in Orested's discovery.

Maxwell's equation still usually believed to be the beginning point of the age of radio waves, as Maxwell showed in theoretical and mathematical form in 1864 that electromagnetic wave could propagate through free space, where the multiple of permittivity and permeability in this equation assures Maxwell's idea about the so called electromagnetism. Hertz in 1888 confirmed Maxwell theory, and Marconi began building a commercial system based on Hertzian waves (radio waves ) that could work on long distance. In spite of all of that, and for our surprise, the analytical form of Maxwell equation shows us something differs from its surface, where we find the electron with its well known mass in kilogram moving with the speed of light when it is at $2.8 \times 10^{-15} \mathrm{~m}$. from another charged particle as follows

$$
c^{2}=\frac{e^{2}}{4 \pi r m_{e} \varepsilon_{0}}
$$

Here $r$ is the nuclear diameter as the distance between the electron and the other charged particle, that caused the electron to move with the speed of light $\mathrm{c}$. Therefore this equation speaks about only one electron and this is far from radio wave propagation, in addition to that while the surface of Maxwell's equation has the so-called electromagnetic appearance, its depth has the nuclear property. No doubt now the success in dealing with radio wave was in dealing practically with excited electrons in atoms striking other electrons causing them to move with the same speed, and through this paper we know that these waves are those of the ocean of coronal electrons in the last layer Sun's structure in which our Earth is immersed.

\section{Conclusion :}

1- The electron is the unit of light as it is shown in the analytical form of Maxwell's equation, and light takes place when electrons are freed from their atoms striking other free electrons in the ocean of electrons of corona over helming the Earth, where radio waves are created, and therefore light does not travel in vacuum.

2- The beginning point of radio waves is not Maxwell's celebrated equation although again this equation proved the electron as the unit of light. Thomas Edison is the discoverer of radio waves those he described as "etheric force"

3- In this paper, radio waves are the waves of the ocean of free electrons in the solar corona over helming the Earth when stroked by electrons freed from their atoms.

\section{References:}

1- www.bibliotecapleyades.net/cienca-so101.html

2- http://www. Space.com117160.sun

3- https://spaceplace.nasa.gov/sun-corona/en

4- http://www.sciencedirect.com/..../

5- Eid Salah : Electronic Explanation for Sun's Radiation and Seasons, Journal of Harmonized Research in Applied Sciences (JOHR).2(1),2014,01-04

6- http://an.m Wikipedia.org/....)History- of-

7- www.einstein- online.info /..../geometry

8- http://www.wikipedia.org /..../History-of-

9- Previous reference

10- www.rare newspapers.com/view/ 633489 